\title{
The Use of Quick Response (QR) Codes in Landmark-Based Pedestrian Navigation
}

\author{
Anahid Basiri, ${ }^{1}$ Pouria Amirian, ${ }^{2}$ and Adam Winstanley ${ }^{2}$ \\ ${ }^{1}$ Nottingham Geospatial Building, Nottingham Geospatial Institute, The University of Nottingham, Innovation Park, \\ Triumph Road, Nottingham NG7 2TU, UK \\ ${ }^{2}$ Department of Computer Science, National University of Ireland, Maynooth, County Kildare, Ireland
}

Correspondence should be addressed to Anahid Basiri; anahid.basiri@nottingham.ac.uk

Received 19 November 2013; Revised 13 March 2014; Accepted 14 March 2014; Published 23 April 2014

Academic Editor: Woojin Chung

Copyright (C) 2014 Anahid Basiri et al. This is an open access article distributed under the Creative Commons Attribution License, which permits unrestricted use, distribution, and reproduction in any medium, provided the original work is properly cited.

\begin{abstract}
Vehicle navigation systems usually simply function by calculating the shortest fastest route over a road network. In contrast, pedestrian navigation can have more diverse concerns. Pedestrians are not constrained to road/path networks; their route may involve going into buildings (where accurate satellite locational signals are not available) and they have different priorities, for example, preferring routes that are quieter or more sheltered from the weather. In addition, there are differences in how people are best directed: pedestrians noticing landmarks such as buildings, doors, and steps rather than junctions and sign posts. Landmarks exist both indoors and outdoors. A system has been developed that uses quick response (QR) codes affixed to registered landmarks allowing users to localise themselves with respect to their route and with navigational instructions given in terms of these landmarks. In addition, the system includes images of each landmark helping users to navigate visually in addition to through textual instructions and route maps. The system runs on a mobile device; the users use the device's camera to register each landmark's QR code and so update their position (particularly indoors) and progress through the route itinerary.
\end{abstract}

\section{Introduction}

Location-based services (LBSs) are now widely used by mobile users in many situations, such as finding their current location on the map or listing locations of nearby shops or facilities. One of the most important LBSs is navigation services which provide users with instructions to get to their destinations, among other functions. Existing car navigation systems are not fully suitable for the navigational needs of pedestrians because car navigation systems are not fully compatible with pedestrians' demands and characteristics. In many cases pedestrians need seamless indoor and outdoor way-finding assistance [1]. The pedestrian navigation system needs other reliable and accurate positioning techniques where globe navigation satellite systems (GNSS) signals are not available. Pedestrians can easily go into buildings as well as streets so an ideal navigation system should work seamlessly in and out of doors. Seamless indoor and outdoor navigation is one of the most important features which should be handled in a pedestrian navigation application and is still a topic of many research projects [2-6].

Another aspect of pedestrian navigation differing from car navigation is non-turn-by-turn navigational instruction delivery. As stated before, in contrast with drivers, pedestrians have a higher degree of freedom in their movements. They are not constrained by road networks (e.g., vehicle lanes, turn restrictions, one-way streets) and can walk in places where vehicles are not allowed to move. They can walk across open areas such as squares, parks, grasslands, or pedestrian malls, which can be traversed freely in any direction [7]. As current turn-by-turn navigational instructions as given to vehicle's drivers are mostly based on graph-based or street networkbased algorithms, this way of navigating is not fully suitable for pedestrians as they do not solely move on streets [8].

Another characteristic of pedestrian movement is the lower speed, in comparison with vehicles. This makes it possible and desirable to provide more detailed information about their path and also the surrounding area they are 
passing through. Such information may make them sure they are on the actual calculated path and have not strayed. Also such information can be applied for tourist guide purposes.

For all the identified challenges for usable pedestrian navigation systems, the use of landmarks can help. It is possible to calculate the position of a user relative to landmark positions. The relative position of the user's device can be sensed using ultrasound [9], dead-reckoning [10], collaborative positioning techniques [11], or inertial sensors [12]. There is also some related research and solutions regarding landmark extraction using laser scan data $[13,14]$. Since landmarks can be detected and labeled both indoors and outdoors (Figure 1), it is possible to determine positions of users seamlessly [15] whether indoors or outdoors.

In addition, it is possible to provide users with navigational instructions landmark-by-landmark rather than turnby-turn. This way of navigating is called landmark-based navigation [16]. Landmark-based navigation is a kind of navigation service in which users are provided with navigational instructions, such as "turn right," "go straight," "turn left," and so forth, whenever they approach each landmark [17]. One of the most important advantages of landmark-based navigation is ensuring the pedestrian that they are on the correct route and not lost, since they are seeing the very landmark which was used as a part of navigational instruction. Also this approach is more compatible for tourists and visitors. It is possible to add some attribute data or even images of landmarks while navigating, so they will observe and learn about an area while being directed [1].

In order to implement a landmark-based navigation service, the Maynooth campus of National University of Ireland was considered. Buildings and important features, such as statues, historic monuments, and the library, were stored in a spatial database as outdoor landmarks. In addition, indoor landmarks such as main entrances and geotagged signs were stored as indoor landmarks. For each landmark, a quick response QR code was generated and affixed. Users can install a QR code reader app and scan the generated code to have access to positional and also other pieces of information. Users' locations can be calculated using either GPS or the QR code approach. Because of availability of GPS signals, it is recommended to apply GPS for outdoor localisation. However, for indoors QR codes are used. Users can scan and use QR codes to localise themselves.

After finding the user location, using GPS for outdoors and QR code positioning for indoors and outdoors, the best path can be calculated and user is given the navigational instructions attaching text and pictures of nearest landmark on the way. Landmarks can be found both indoors and outdoors so this approach can provide navigation services seamlessly and also pedestrians see an image of nearest landmark so they can enjoy visiting the campus by getting information about each building while moving.

In this paper we describe the design and implementation of a system that uses landmarks using QR codes as the main method of pedestrian routing. Landmark-based navigation and related concepts are explained in Section 2. Section 3 is focused on implementation and evaluation. The conclusions in Section 4 describe future directions for pedestrian navigation.

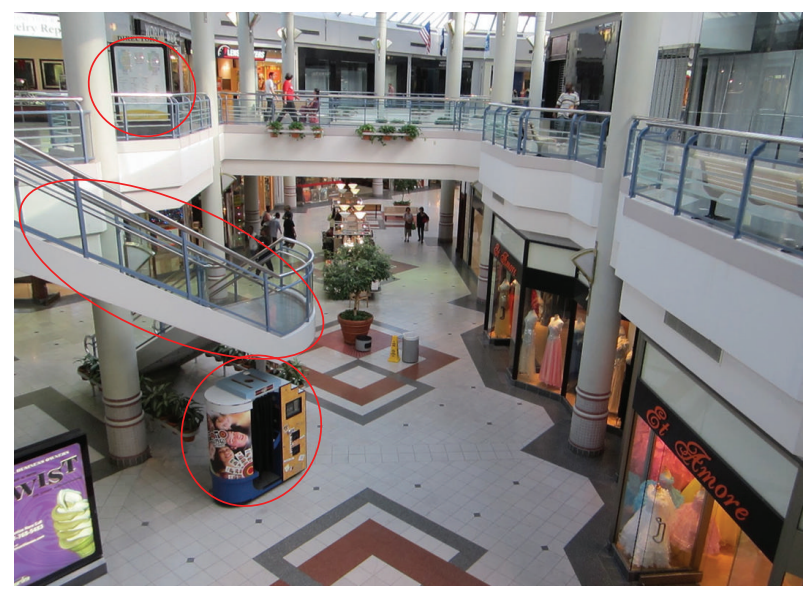

FIGURE 1: Indoor landmarks.

\section{Landmark-Based Pedestrian Navigation}

A pedestrian has several possible navigational strategies to find a desired destination or goal [18].

(1) The individual has no information and is forced to search randomly (random navigation).

(2) The individual moves towards a visible cue which leads to the arrival point (taxon navigation).

(3) The individual follows a fixed motor program (praxic navigation, e.g., "turn left after 200 meters, then turn right after 150 meters").

(4) The individual associates directions with visual cues (route navigation, e.g., "turn left at the church").

(5) The individual forms a mental representation of the surroundings and is able to plan routes between any locations within the area (locale navigation).

Landmarks can have an important role in random navigation, taxon navigation, route navigation, and local navigation. Several researchers in the field of spatial cognition assert that navigating humans rely on three forms of spatial knowledge: landmark, route, and survey knowledge $[19,20]$. Exploring an unfamiliar environment, pedestrians first notice outstanding objects or structures at fixed locations. These unique objects or places are easy to recognize and can be kept in memory without difficulty [15].

This shows the importance of the meaning of landmarks for human navigational tasks. Landmarks are stationary, distinct, and salient objects or places, which serve as cues for structuring and building a mental representation of the surrounding area. Any object can be perceived as a landmark if it is unique enough in comparison to the adjacent items. The importance of landmarks for pedestrian navigation and way-finding instructions has been proved and demonstrated [21-24].

In this section landmark and landmark-based navigation concepts and related definitions are explained. Landmarks should be stored in a database, and then it becomes possible to localize users or provide them with landmark-based 
navigational instructions. The first definition and attributes of landmarks are discussed in more detail. Then the two important components of a landmark-based navigation service are explained: landmark-based positioning and path finding.

2.1. What Is a Landmark? A landmark can be defined as anything which is easily recognizable, such as a monument or a building. Landmarks are particularly interesting to tourists probably due to notable physical features or their historical significance. Landmarks are often used in casual navigation by ordinary people, such as giving directions.

In urban studies as well as in geography, a landmark is furthermore defined as an external point of reference That helps people to localise, familiarise, and also remember the environment [25]. Landmarks are also used in verbal route instructions. These two properties of landmarks are very important and potentially helpful in navigation systems and services. In general, location of a landmark is fixed since usually landmarks are not moving objects. So it is possible to measure, store, and use landmarks' position for localisation purposes. Pedestrians can localise themselves by finding their relative location with respect to a landmark and finally calculate their absolute position.

Landmarks should be carefully chosen in order to be easy to identify; for example, a large building should have priority over a small one. A feature which has sufficient contrast to the background is a good option to be considered as landmark since its image would be recognizable to users. Such objects have to possess a certain saliency, which makes them remarkable and distinctive. So the surrounding area influences the characteristics a point must have to be perceived as a landmark (e.g., a shopping center may not be very outstanding in urban areas but becomes a salient landmark when being situated in a rural village). In Section 3, the process of landmark extraction and storage in a spatial database is explained in more detail.

After extracting important features as landmarks, the optimal path between the current location of the user and their selected destination should be found. This path is then used to generate navigational instructions. The next subsection focuses on landmark-based localization. Then a landmark-based path-finding algorithm is explained.

2.2. Landmark-Based Positioning and Path Finding. In general, there are two main categories of landmark-based positioning techniques: image-based and non-image-based. Two examples of image-based landmark positioning are using QR codes and through image-matching. The location of the user is calculated using image processing techniques such as feature extraction and feature matching against a database of landmark images. The calculation can be done on the mobile device itself or a remote server. Another imagebased positioning approach is using ceiling-mounted cameras which can identify people and track their location. However, this needs additional infrastructure in comparison with methods using the mobile device's sensors.

Non-image-based positioning techniques such as radio frequency identification (RFID) and bluetooth network positioning usually need an additional chipset fitted into the mobile device, such as RFID readers, Bluetooth devices, and so forth, to get signals and calculate the position.

This paper focuses on positioning techniques using QR codes which have a number of advantages over other methods. Mobile phones are equipped with the cameras to read QR codes as standard. In addition, QR codes can provide a position directly using a reader application and there is no need to have complicated computations. The mobile device will in consequence consume less power in comparison with other positioning techniques. In addition, users only scan the QR codes whenever they need to ascertain their position, helping save power over other approaches that usually need to have the relevant receiver on (Bluetooth, RFID, GPS, etc.) constantly. QR codes have the added advantage over camera network-based positioning in the preservation of the privacy of users. QR-code-based positioning is a push service rather than a pull service - the user and application ask for a position whenever needed rather than being constantly tracked.

Registered landmarks and their precise positions are stored in a spatial database. In addition to landmark's position, some nongeometrical information such as age, type, etc. is stored in the data base. Also there are at least four images from each landmark to show it from different angles. These images will be used in image-based navigational instructions. Then for all landmarks unique QR codes encoding the precise location of each landmark are generated. It is also possible to encode some additional information about the landmark, such as name, age, a link to images of the landmark stored in the database or available on the web (URL), and so forth; such information can help as a part of navigational instruction or as a tourist guidance module.

Generated and printed QR codes are affixed to each landmark. Mobile phone users are able to access data quickly through the use of QR codes. To use a QR code, one would need a smart-phone, iPod, notebook or similar device with a camera, a QR code reader application installed, and, in most cases, Internet access, either through a wireless network or through a data phone plan. The user would scan the code and gain access to a variety of information, including the location of the landmark. Since the absolute positions of landmarks are encoded, it is possible to assign the same coordinates to the user's position. Although this approach may not provide the most accurate position, the level of inaccuracy can be ignored considering all resources of uncertainty in other positioning techniques such as multipath in GNSS, line of sight in RFID, and Wi-Fi positioning. In addition, the user usually will find and scan the QR code tags when they are very close to the landmark so taking landmark's position as user's position is not a very incorrect assumption. After finding user's position, it is used in path finding and navigational services.

In order to find the shortest path between two points, path-finding algorithms try to minimize the cost of the journey, usually in terms of distance or travel time, although other costs may also be applied such as how exposed a route is to weather. The campus navigation system is designed to provide a service to pedestrians so the shortest and the fastest path algorithms usually have the same outputs. We put another option for path finding which calculates the most reliable path. Most of the time users want to get navigational 
instruction if they do not know the place very well. In the case of not being familiar with the environment, usually users are looking for the most reliable path rather than the shortest one. Taking the most reliable path may reduce the chance of getting lost. In addition, most users of a tourist navigation application want to see monuments and landmarks which may need deviation from the shortest path. Landmark-based path-finding algorithm is providing a more attractive and, at the same time, a more reliable path. Since users are seeing more landmarks on the way, they get less bored and also they are surer that they are taking the right route.

In landmark-based path-finding we look for a path Which maximises number of landmarks while minimising distance to be traversed. So a landmark-based path-finding algorithm tries to minimize the result of

$$
\frac{\text { length of edge }}{\text { number of landmarks on edge }} \text {. }
$$

Using this edge cost, the route is calculated with the minimum total cost. The route is then presented to the user using images and textual descriptions of landmarks that can be viewed along this route. The next section describes the implementation of this positioning and path-finding method.

\section{Implementation}

The prototype landmark-based navigation system is intended to provide navigation services to pedestrians in the campus of National University of Ireland Maynooth. There are four main steps in its design. First, landmarks are defined, extracted, and stored in a spatial database. In this step both geometrical and nongeometrical characteristics of each landmark are stored.

The second component determines position (Figure 3). It calculates users' positions using QR code positioning. A third component is the routing algorithm, which captures start point and destination point as two inputs and calculates the least-cost path. Finally, navigational instructions which help users to get to their destination are generated in terms of the landmark information and photos. This section explains these four steps and their implementation and integration into the campus navigation application.

3.1. Landmark Extraction. As stated before, landmarks have to possess a certain saliency which makes them remarkable and distinctive. After selecting some features on the campus as landmarks, they were stored in a spatial database as spatial objects with attributes such as importance, type, label, and name. Landmark extraction and storage can be largely automated using algorithms and approaches discussed before.

In order to identify important features on the campus to be stored in the database as landmarks, three criteria were considered: uniqueness, contrast with surroundings, and size. For example, Figure 2 shows one of the most important landmarks on the NUIM campus, extracted from a 3D model of the campus, which is the highest and the most unique feature which can be seen from most locations.

In order to select landmarks, one of the best approaches is comparing the attribute values of data records (such as

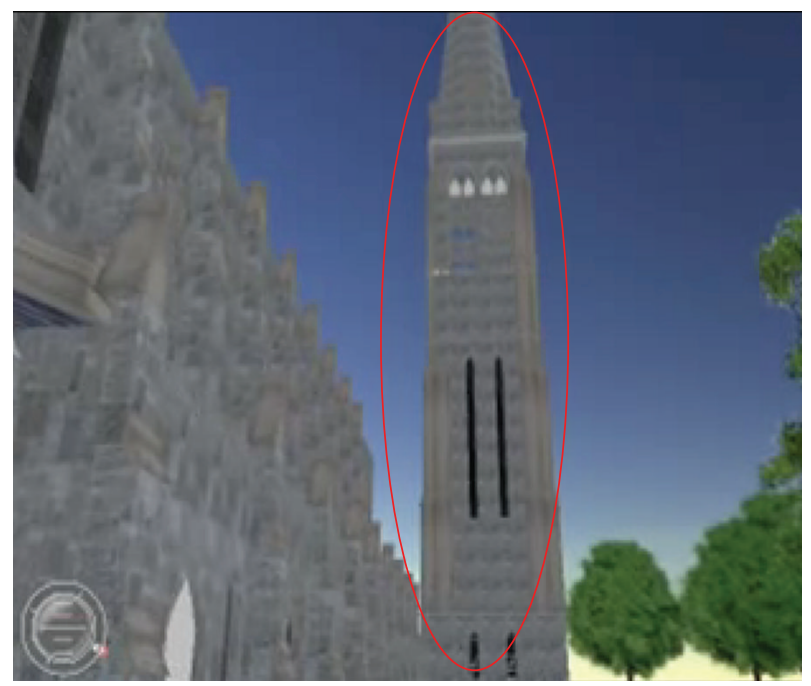

FIgURE 2: Landmark extraction from 3D model of NUIM campus.

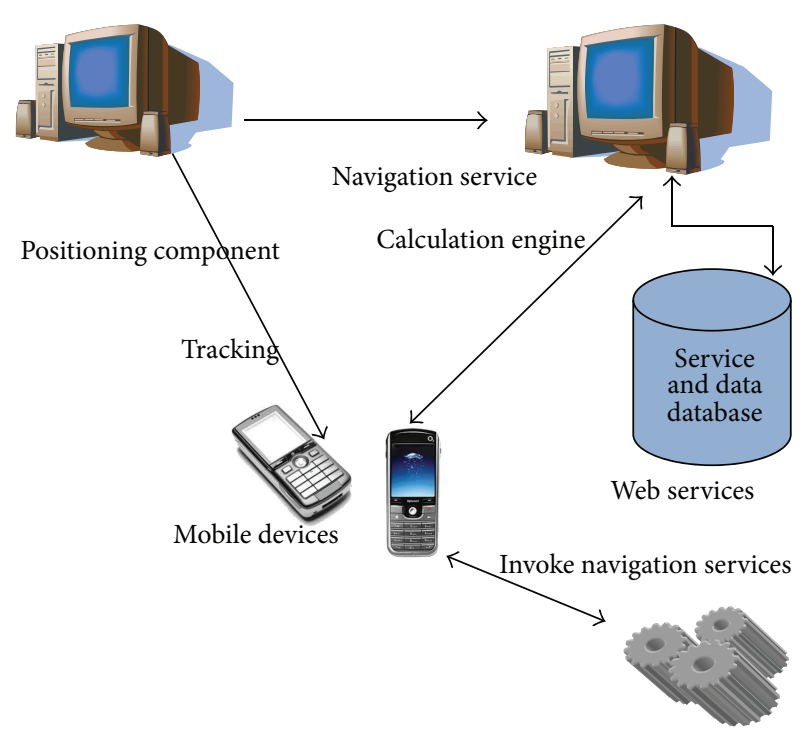

FIGURE 3: NUIM campus landmark-based navigation system architecture.

the geometrical properties of each spatial object, buildings usage, and building labels) and identifying the most suitable landmark by ranking all attributes at potential decision points (as proposed in [26]). The only problem in this approach is it disregards the fact that the visibility of an object is not invariable and that the quality of a landmark can differ depending on the direction of view [27]. In order to solve this issue, the visibility of each feature can be calculated using a digital surface model (DSM). A DSM of the campus is available from the 3D campus project. Features, which can be viewed from far distances, are considered as suitable landmarks based on which we can provide landmark-based navigational instruction.

Knowing the height of the user and the height of each candidate landmark, it is possible to calculate if the landmark is visible from the user's point of view. Seeing the majority of 
a landmark increases the certainty that the user is on the correct route. Formula (2) shows the percent of visibility of an object. If there is no intervening object that acts as an obstacle, then you can see the whole landmark and if the intervening object is as high as the landmark you cannot see it. $\left(L_{2}-H\right) / L_{2}$ is a factor which shows percent of visibility of a landmark,

$$
\frac{\left(L_{2}-H\right)}{L_{2}}=\frac{\left(D_{1} * L_{2}+D_{2} * L_{1}\right)}{\left(D_{1}+D_{2}\right)}+1 .
$$

In formula (2), $L_{1}$ is the user's height and $L_{2}$ is height of the landmark to be seen. $H$ shows the height of the intervening object and $D_{1}$ shows distance between user and intervening object. $D_{2}$ is distance between intervening object and the landmark to be seen.

Assuming $L_{1}$ is approximately 160 centimeters, the average human height, it is possible to calculate visibility of each landmark from the user's point of view. This is used to select landmarks which can be seen from users' point of view along the route.

3.2. Landmark-Based Navigation. Using landmarks stored in a spatial database, The optimal route can be calculated and landmark-based navigational instructions can be provided to users. The architecture used to provide the user with landmark-based navigational instructions is illustrated in Figure 3. The landmark-based navigation system implemented as part of the eCampus system in National University of Ireland Maynooth consists of four main components: a positioning component, a landmark database, the navigation service calculation engine, and an application running on the user's mobile device.

The positioning component is responsible of getting the position of the user which can be provided by GPS or QR codes. The navigation service calculation engine uses the user's position as input to two other services. First it calculates the optimal route using user's position and selected destination and secondly it calculates visible landmarks on the way. In order to do both of these tasks, the navigation service calculation engine needs to have access to the spatial database where landmarks' information, such as location and size, is stored. In this project, a graph database is used.

In general, elements in landmark-based navigation services can change frequently, since a landmark can be visible to one user but not another. Processing of such highly connected and interrelated data can be problematic for most conventional storage systems such as relational database management systems (RDBMS) [28]. By their nature, navigation services need to be highly responsive in real time (or near real time). In addition, since network-based systems generally need to service a huge number of users, such services must provide appropriate scalability and performance $[29,30]$. In addition, many network-processing systems preanalyse and store the results along with the network data itself. However, when additional network elements (such as new landmarks or new roads) have to be added to the data, all the representation and built network must be rebuilt and recompiled. This recompilation process would be a serious processing



FIGURE 4: Suggested route based on landmark-based path-finding algorithm.

task especially when the newly added elements are highly connected to the existing elements. In order to avoid this, it is better to store topologically connected data in their natural representation. The graph database is suitable for this [29].

The navigation service calculation engine uses both landmark and network edge-node data to determine the optimal path. Figure 4 shows an example route (from the NUIM south campus main entrance to Callan Building room number 1.38). Some parts of the calculated route pass inside buildings, such as end segments inside the Callan Building. In order to determine the user's position inside the building, QR codes are used.

Following the route calculation stage, the navigational instructions must be communicated to the user. In this step, the navigation service calculation engine uses the route topology also in conjunction with landmark data stored in the database to determine the landmarks to be seen from the proposed route. As the user proceeds and the location changes (including when a new QR code is scanned by user) this process will be repeated and a new set of navigational instructions with landmarks will be provided.

It is also possible to provide users with images of landmarks on the way, and, if required, give them more information about the history and other attributes of the building. In Figure 5, an example route is shown in the application. As illustrated, five landmarks are detectable on the calculated route, both indoors and outdoors. At each landmark, related information is delivered to the user.

Since the navigational instructions contain information about landmarks which can be seen along the route, this approach may be more suitable in tourist navigation applications. In addition, users feel more secure from getting lost or straying from the route as they are provided with the pictures of landmarks which they can see as they proceed.

Figure 6 shows the web application interface of the system which provides images of landmarks as a part of its navigation service. Three modes of travel: pedestrian, vehicle, 


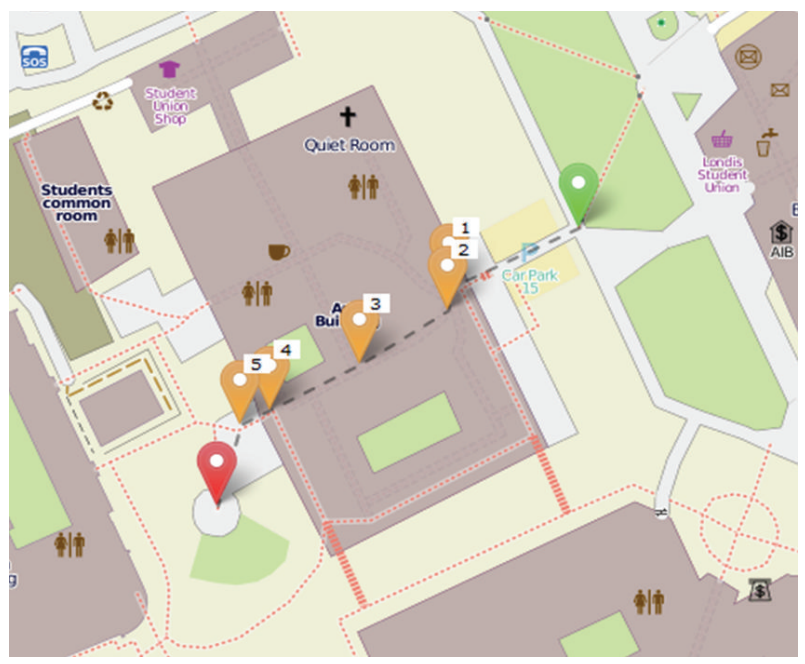

FIGURE 5: A route with five landmarks on the way.

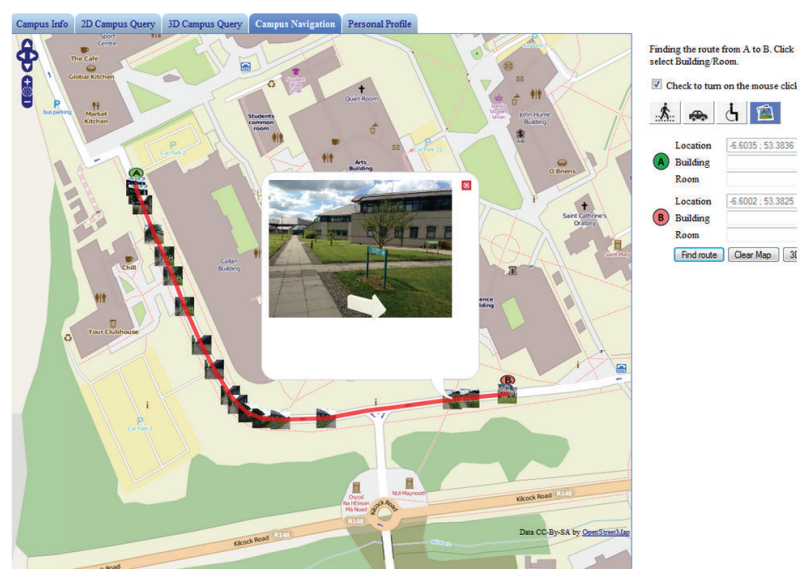

FIgURE 6: Navigation services provided in the eCampus web application.

and wheelchair can use this service. The web application is available on http://147.252.87.12/eCampTest/Routing/.

\section{Conclusion}

Landmarks are often used for casual navigation, such as giving directions, by ordinary people. In this paper we have demonstrated that this can be implemented in navigation applications as well as to provide users with landmark-based navigational instruction. The positions of users can be calculated in relation to the nearest landmark either through object recognition or the use of QR codes fixed to objects. Then, based on the destination selected by the user interactively, a landmark-based path-finding algorithm, which calculates a route with maximum landmarks on the way and a minimum distance, calculates the optimal path. Users can be provided with information about interesting or important features, landmarks, on the route to make sure they are on the correct way as well as for general interest. This paper explains these three steps in detail and the implementation of a landmarkbased navigation application.

\section{Conflict of Interests}

The authors declare that there is no conflict of interests regarding the publication of this paper.

\section{Acknowledgments}

Research presented in this paper was funded by a Strategic Research Cluster Grant (07/SRC/I1168) by Science Foundation Ireland under the National Development Plan. The authors gratefully acknowledge this support. This work was financially supported by EU FP7 Marie Curie Initial Training Network MULTI-POS (Multitechnology Positioning Professionals) under Grant no. 316528.

\section{References}

[1] A. Basiri, A. C. Winstanley, and P. Amirian, "Landmark-based pedestrian navigation," in Proceedings of the 21st GIS Research (GISRUK) Conference, 2013.

[2] J. Cheng, L. Yang, Y. Li, and W. Zhang, "Seamless outdoor/indoor navigation with WIFI/GPS aided low cost Inertial Navigation System," in Physical Communication, 2014.

[3] T. Zhang and X. Xu, "A new method of seamless land navigation for GPS/INS integrated system," Measurement, vol. 45, no. 4, pp. 691-701, 2012.

[4] X. Li, J. Wang, and T. Li, "Seamless positioning and navigation by using Geo-referenced images and multi-sensor data," Sensors, vol. 13, no. 7, pp. 9047-9069, 2013.

[5] R. Hansen, R. Wind, C. S. Jensen, and B. Thomsen, "Seamless indoor/outdoor positioning handover for location-based services in streamspin," in Proceedings of the 10th International Conference on Mobile Data Management: Systems, Services and Middleware, MDM 2009, pp. 267-272, May 2009.

[6] A. Basiri, A. Winstanly, P. Amirian, M. Sester, and C. Kuntzsch, "Uncertainty handling in navigation services using rough and fuzzy set theory," in Proceedings of the International Workshop on Querying and Mining Uncertain Spatio-Temporal Data (ACM SIGSPATIAL QUeST '12), Redondo Beach, Calif, USA, 2012.

[7] C. Gaisbauer and A. U. Frank, "Wayfinding model for pedestrian navigation," in Proceedings of the AGILE International Conference on Geographic Information Science, 2008.

[8] M. Pielot and S. Boll, "“'In fifty metres turn left": why turnby-turn instructions fail pedestrians," in Proceedings of the Workshop on Using Audio and Haptics for Delivering Spatial Information via Mobile Devices, Lisboa, Portugal, September 2010.

[9] S. Holm, "Hybrid ultrasound-RFID indoor positioning: combining the best of both worlds", in Proceedings of the IEEE International Conference on RFID (RFID '09), pp. 155-162, Orlando, Fla, USA, April 2009.

[10] A. S. Etienne and V. Seguinot, "Navigation by dead reckoning and local cues," Journal of Navigation, vol. 46, no. 3, pp. 364370, 1993.

[11] J. K. Lee, D. A. Grejner-Brzezinska, and C. Toth, "Networkbased collaborative navigation in GPS-denied environment," Journal of Navigation, vol. 65, pp. 445-457, 2012. 
[12] R. Vepa, "Ambulatory position tracking of prosthetic limbs using multiple satellite aided inertial sensors and adaptive mixing," Journal of Navigation, vol. 64, no. 2, pp. 295-310, 2011.

[13] P. Núñez, R. Vázquez-Martín, A. Bandera, and F. Sandoval, "Fast laser scan matching approach based on adaptive curvature estimation for mobile robots," Robotica, vol. 27, pp. 469-479, 2009.

[14] C. F. Chang, C. C. Tsai, J. C. Hsu, S. C. Lin, and C. C. Lin, Laser Self-Localization for a Mobile Robot Using Retro-Reflector Landmarks, Department of Electrical Engineering, National Chung Hsing University, 2005.

[15] A. Millonig and K. Schechtner, "Developing landmark-based pedestrian navigation systems," in Proceedings of the 8th International IEEE Conference on Intelligent Transportation Systems, pp. 197-202, September 2005.

[16] A. J. May, T. Ross, and S. H. Bayer, "Incorporating landmarks in driver navigation system design: an overview of results from the REGIONAL project," Journal of Navigation, vol. 58, no. 1, pp. 4765, 2005.

[17] Z. Fang, Q. Li, X. Zhang, and S. L. Shaw, "A GIS data model for landmark-based pedestrian navigation," International Journal of Geographical Information Science, vol. 26, no. 5, 2012.

[18] D. Redish, Beyond the Cognitive Map: From Place Cells to Episodic Memory, MIT Press, Cambridge, UK, 1999.

[19] A. W. Siegel and S. H. White, "The development of spatial representations of large-scale environments," Advances in Child Development and Behavior, vol. 10, pp. 9-55, 1975.

[20] S. Werner, B. Krieg-Brückner, H. Mallot, K. Schweizer, and C. Freksa, "Spatial cognition: the role of landmark, route and survey knowledge in human and robot navigation," in Informatik Aktuell, M. Jarke, K. Pasedach, and K. Pohl, Eds., pp. 4150, Springer, Berlin, Germany, 1997.

[21] P. E. Michon and M. Denis, "When and why are visual landmarks used in giving directions?" in Spatial Information Theory, pp. 292-305, Springer, Heidelberg, Germany, 2001.

[22] T. M. Denis, "Referring to landmark or street information in route directions: what difference does it make?" in Spatial Information Theory, W. Kuhn, M. Worboys, and S. Timpf, Eds., vol. 2825 of Lecture Notes in Computer Science, pp. 384-397, Springer, Heidelberg, Germany, 2003.

[23] K. Lovelace, M. Hegarty, and D. Montello, "Elements of good route directions in familiar and unfamiliar environments, in spatial information theory," C. Freksa and D. M. Mark, Eds., pp. 65-82, Springer, Heidelberg, Germany, 1999.

[24] M. Raubal and S. Winter, "Enriching wayfinding instructions with local landmarks," in Geographic Information Science, M. J. Egenhofer and D. M. Mark, Eds., vol. 2478 of Lecture Notes in Computer Science, pp. 243-259, Springer, Heidelberg, Germany, 2002.

[25] K. Lynch, The Image of the City, MIT Press, 1960.

[26] B. Elias, "Determination of Landmarks and Reliability Criteria for Landmarks," in Proceedings of the 5th Workshop on Progress in Automated Map Generalization, IGN , Paris, France, 2003.

[27] B. Brenner and B. Elias, "Extracting landmarks for car navigation systems using existing GIS databases and laser scanning," in Proceedings of the ISPRS Workshop on Photogrametric Image Analysis, Munich, Germany, 2003.

[28] P. Amirian, A. Winstanley, and A. Basiri, NoSQL Storage and Management of Geospatial Big Data With Emphasis on Serving Geospatial Data Using Standard Geospatial Web Services, GIS Research (GISRUK), University of Liverpool, Merseyside, UK, 2013.
[29] P. Amirian, A. C. Winstanley, and A. Basiri, "Using graph databases in LBS applications: storing and processing navigational and tracking data," Proceedings of the Mobile Gehnt Conference, 2013.

[30] P. Amirian, A. Basiri, and A. Alesheikh, "Standard-based, Interoperable Services for Publishing Urban Services Data," Elsevier Computers, Environment and Urban System, vol. 34, no. 4, pp. 309-321, 2010. 

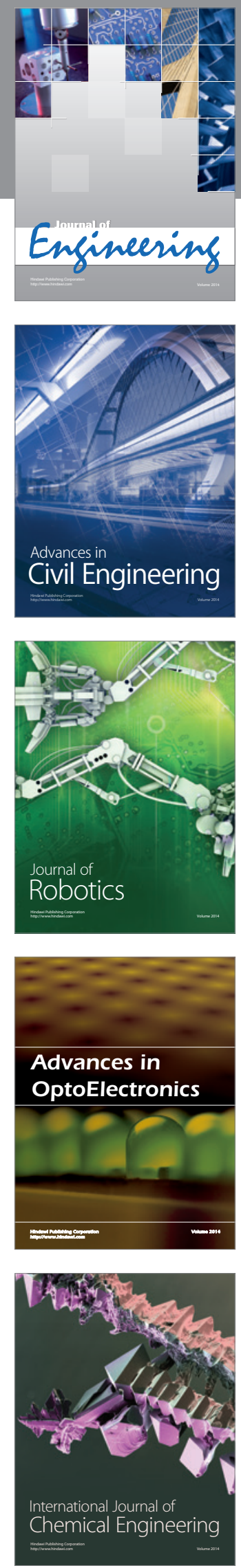

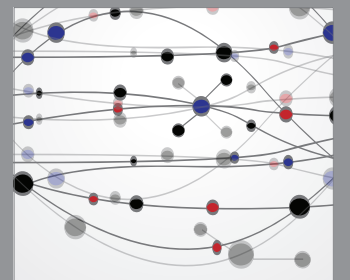

The Scientific World Journal
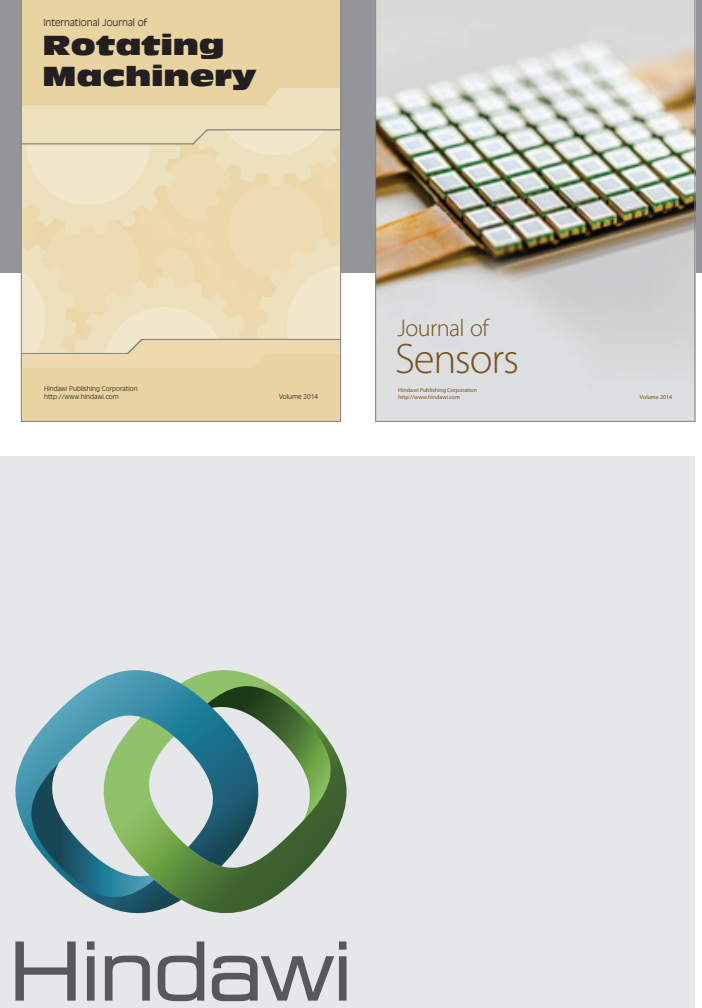

Submit your manuscripts at http://www.hindawi.com

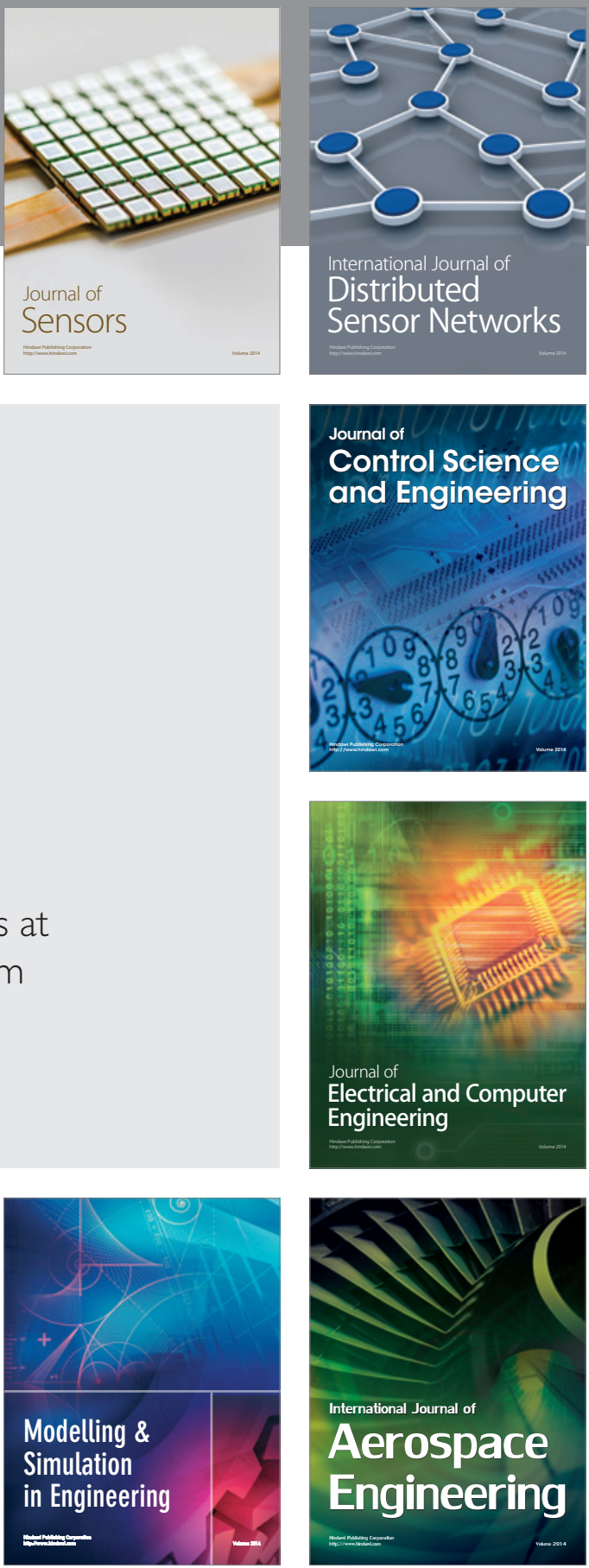

Journal of

Control Science

and Engineering
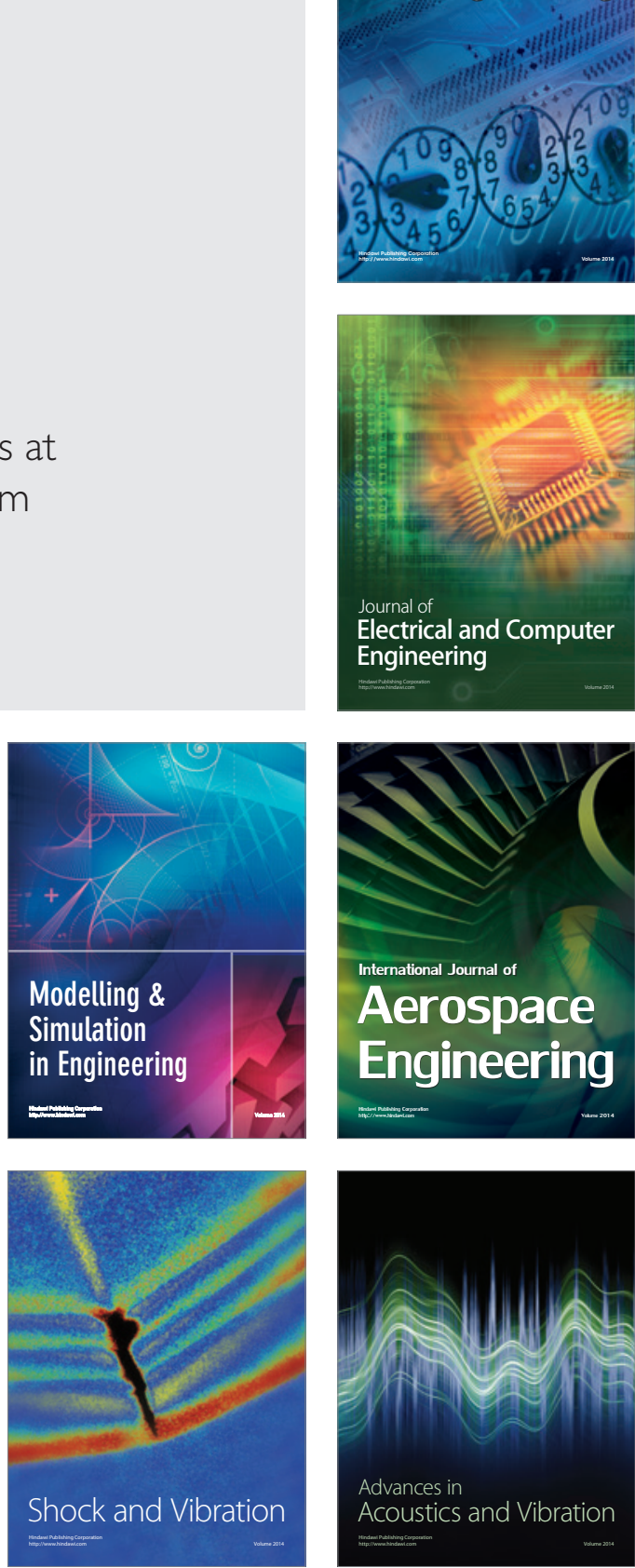